\title{
Profissionais de enfermagem de um serviço de urgência e emergência frente ao suicídio na adolescência
}

\author{
Nursing professional from and emergency service in front of suicide in adolescence \\ Professionales de enfermería de un servicio de emergencia frente al suicidio en \\ adolescencia
}

Liliane de Lourdes Teixeira Silva ${ }^{1 *}$, Bianca Penido Vecchia ${ }^{1}$, Thiago Magela Ramos², Thaís Aparecida Faria Costa ${ }^{1}$.

\section{RESUMO}

Objetivo: O presente estudo objetivou compreender como os profissionais de enfermagem de um serviço de urgência e emergência percebem o suicídio na adolescência. Métodos: Trata-se de uma pesquisa com abordagem qualitativa. Para coleta de dados, ocorrida em fevereiro de 2016, foi utilizado roteiro de entrevista semi-estruturado elaborado pelos próprios autores. Fizeram parte do estudo 12 enfermeiros e 12 técnicos de enfermagem que atuam em uma Unidade de Pronto Atendimento. Os dados obtidos foram analisados de acordo com a análise de conteúdo de Bardin. Resultados: Duas categorias emergiram da análise de dados: "O mundo externo e a tentativa de suicídio na adolescência" e "O mundo interno e a tentativa de suicídio na adolescência". A assistência ao adolescente que tenta o suicídio baseia-se prioritariamente a cuidados técnicos e empíricos. Mitos quanto a tentativa de suicídio emergiram das falas dos profissionais. Conclusão: Percebe-se a necessidade de educação permanente com os profissionais que atuam diretamente nos serviços e urgência emergência e que atendem o adolescente que tenta suicídio. Ressalta-se a necessidade da atuação de uma equipe multiprofissional que possa analisar e oferecer uma assistência plena ao adolescente.

Palavras-chave: Suicídio, Adolescência, Enfermagem.

\begin{abstract}
Objective: This study aimed to understand how nursing professionals in an urgent and emergency service perceive suicide in adolescence. Methods: This is a research with a qualitative approach. For data collection, which took place in February 2016, a semi-structured interview script prepared by the authors themselves was part of the study. 12 nurses and 12 nursing technicians who work in an Emergency Care Unit. The data obtained were analyzed according to Bardin's content analysis. Results: Two categories emerged from the data analysis: "The external world and the adolescent suicide attempt" and "The internal world and the adolescent suicide attempt". Assistance to adolescents who attempt suicide is primarily based on technical and empirical care. Myths about the suicide attempt emerged from the professionals' statements. Conclusion: The need for permanent education is perceived with the professionals who work directly in the emergency services and who attend the adolescent who tries to commit suicide. The need for a multidisciplinary team to analyze and offer full assistance is emphasized. to the teenager.
\end{abstract}

Key words: Suicide, Adolescence, Nursing.

\section{RESUMEN}

Objetivo: Este estudio tuvo como objetivo comprender cómo los profesionales de enfermería en un servicio de urgencias y emergencias perciben el suicidio en la adolescencia. Métodos: Esta es una investigación con un enfoque cualitativo. Para la recopilación de datos, que tuvo lugar en febrero de 2016, fue parte del estudio un guión de entrevista semiestructurada preparado por los propios autores.12 enfermeras y 12 técnicos de

1 Universidade Federal de São João del Rei (UFSJ), Divinópolis - MG. *E-mail: lilanets@ufsj.edu.br

2 Prefeitura Municipal de Carmo do Cajuru, Carmo do Cajuru - MG.

SUBMETIDO EM: 5/2020

ACEITO EM: 6/2020

PUBLICADO EM: 9/2020 
enfermería que trabajan en una Unidad de Atención de Emergencia. Los datos obtenidos se analizaron según el análisis de contenido de Bardin. Resultados: Del análisis de datos surgieron dos categorías: "El mundo externo y el intento de suicidio en adolescentes" y "El mundo interno y el intento de suicidio en adolescentes". La asistencia a los adolescentes que intentan suicidarse se basa principalmente en la atención técnica y empírica. Los mitos sobre el intento de suicidio surgieron de las declaraciones de los profesionales. Conclusión: La necesidad de educación permanente se percibe con los profesionales que trabajan directamente en los servicios de emergencia y que atienden al adolescente que intenta suicidarse, y se destaca la necesidad de un equipo multidisciplinario que pueda analizar y ofrecer asistencia completa al adolescente.

Palabras clave: Suicidio, Adolescencia, Enfermería.

\section{INTRODUÇÃO}

O suicídio consiste na ação de pôr fim a própria vida em um ato deliberado, executado de forma consciente e intencional. O Brasil constitui o oitavo país em número absoluto de suicídios. Em 2016, foram registradas 13.467 mortes no país, sendo 10.203 homens e 3.263 mulheres (WORLD HEALTH ORGANIZATION, 2019).

Devido às mudanças e adaptações características da adolescência, como as transformações biológicas da puberdade e aquelas relacionadas à maturidade biopsicossocial, esta etapa torna-se um período vulnerável para a ocorrência do suicídio. O suicídio na faixa etária de 15 a 29 anos foi a segunda principal causa de morte entre os homens e a terceira maior causa entre as mulheres (WORLD HEALTH ORGANIZATION, 2019).

A equipe de enfermagem é responsável pelos cuidados iniciais ao paciente que tenta suicídio, além disso, devido à essência de sua atividade, que é o cuidado, permanece por mais tempo em contato direto com o ser assistido. Ao lado destes pacientes quando adolescentes, tal equipe possui potencialidade para auxiliar na prevenção de novas tentativas de suicídio (MORAES SM, et al., 2016).

Sabe-se que as atitudes preconceituosas e judiciosas dos profissionais de saúde frente àqueles que tentaram o suicídio, podem gerar nos pacientes experiências traumáticas, que o levarão a distanciar-se dos serviços de saúde (SOUZA ACG, et al., 2015).

A qualidade do atendimento prestado ao paciente que tenta autoextermínio está diretamente relacionada a atitudes e posturas menos negativas e opressoras frente ao episódio, por parte da equipe que o atende. $\mathrm{Na}$ assistência de enfermagem, atitudes de compreensão e de cuidado frente ao suicídio estão associadas à aquisição de conhecimentos e à reflexão, portanto, podem ser influenciadas pela sua formação acadêmica (MORAES SM, et al., 2016).

A frágil formação dos profissionais de enfermagem para lidar com o processo de morte, principalmente quando o usuário supostamente deseja morrer, no caso da tentativa de suicídio, é evidenciada pela literatura (SANTOS RS, et al., 2017; STORINO BD, et al., 2018).

A formação do profissional enfermeiro baseia-se estruturalmente em salvar vidas, portanto ao se deparar com alguém que tenta o suicídio, principalmente se jovem, o profissional tende a captar apenas o lado agressivo dessa tentativa, desprezando o sofrimento psíquico daquele que demanda cuidados não apenas físicos, mas também morais e psicológicos. Essa atitude pode influenciar no restabelecimento ou não desse adolescente que demanda cuidados (JANUÁRIO JP e ÂNGELO MER, 2017).

Sabendo da importância do manejo da tentativa de suicídio do adolescente pelos profissionais de enfermagem e na capacidade do mesmo em impactar positivamente ou negativamente no comportamento futuro do adolescente, questiona-se: como os profissionais de enfermagem de uma unidade de urgência e emergência percebem o suicídio na adolescência?

Espera-se que com este estudo seja possível identificar as lacunas existentes na relação profissional de enfermagem $x$ adolescente suicida e propor ações que visem a otimizar 0 atendimento as pessoas que tentaram suicídio. Portanto o presente estudo tem como objetivo: compreender a percepção dos enfermeiros e dos técnicos de enfermagem de um serviço de urgência e emergência frente ao suicídio na adolescência. 


\section{MÉTODOS}

Trata-se de um estudo de abordagem qualitativa, realizado em uma Unidade de Pronto Atendimento (UPA) do estado de Minas Gerais. O local do estudo presta atendimento aos usuários do SUS por demanda espontânea e encaminhamentos da rede básica de 54 cidades interligadas por um consórcio. A clientela é composta por pacientes com quadros clínicos graves e atendimentos de urgência e emergência, recebendo constantemente pacientes com conduta suicida.

Foram incluídos no estudo enfermeiros e técnicos de enfermagem que possuíam experiência no atendimento a adolescentes que tentaram suicídio. Inicialmente todos os profissionais de enfermagem foram abordados com o intuito de verificar quais se encaixavam nos critérios de inclusão e em seguida foi realizado o convite para participação na pesquisa. Ao final participaram 24 membros da equipe, sendo 12 enfermeiros e 12 técnicos de enfermagem. As entrevistas foram finalizadas quando os pesquisadores identificaram a saturação dos dados.

As entrevistas foram realizadas no próprio ambiente de trabalho em local privativo e em data e horário marcados pelos participantes. Utilizou-se um roteiro semiestruturado com as seguintes questões norteadoras: "Qual a sua percepção acerca da tentativa de suicídio na adolescência?" e "Na sua opinião, quais as principais causas que levam o adolescente a tentar o autoextermínio?". As entrevistas foram gravadas com autorização dos participantes e, posteriormente, transcritas.

Os dados foram analisados segundo análise de conteúdo de Bardin seguindo as etapas de pré-análise, exploração do material e tratamento dos resultados obtidos e interpretação. Esta última foi realizada utilizando como referência literatura nacional einternacional pertinente sobre o tema.

Para compreensão da percepção este estudo orientou-se pela definição de Kant sobre percepção, conduzida pela colaboração de Lev Vygotsky, onde percepção é um processo ativo, evocado também pela memória e que leva em consideração aspectos biológicos, culturais e históricos. Ou seja, o indivíduo percebe o que lhe é relevante, tornando assim em conhecimento a matéria que ele julga ser importante para sua (sobre) vivência (PALANGANA IC, 2015).

A referida pesquisa foi submetida ao comitê de ética em pesquisa da Universidade Federal de São João del Rei (UFSJ), sendo aprovado sob registro CAAE: 51216515.2.0000.5545 em 11 de fevereiro de 2016. Os dados foram coletados após aprovação do Comitê de ética, ainda no mês de fevereiro de 2016. Na análise e discussão dos dados os enfermeiros foram identificados pela letra $E$ e os técnicos de enfermagem pela letra TE, ambos seguidos de uma numeração.

\section{RESULTADOS}

Após a análise dos dados emergiram duas categorias de análise: "O mundo externo e a tentativa de suicídio na adolescência". Na primeira categoria os entrevistados discursaram sobre a repercussão das relações familiares e amorosas, a falta de apoio social e uso de drogas nas tentativas de suicídio na adolescência. Na segunda categoria, intitulada "O mundo interno e a tentativa de suicídio na adolescência", esses mesmos profissionais discorreram sobre os efeitos das alterações psicossociais da adolescência, dos conflitos internos e do adoecimento mental e sua influência nas tentativas de suicídio.

Primeiramente, há de se destacar que a tentativa de suicídio na adolescência é antes de tudo percebida pelos profissionais de enfermagem, ou seja, é um ato que chama a atenção dos mesmos segundo os relatos coletados. As falas de aumento no número de atendimento estiveram presentes em 05 entrevistas, abaixo destacamos duas:

"[...] não são dados científicos, mas é que está tendo um aumento né? No índice de suicídio entre os jovens, é o que eu percebo é isso aqui na emergência." (E2)

"[...] percebe-se que tem um grande número que a gente atende né? Na urgência e emergência de adolescentes que tentam o autoextermínio, cada vez aumentando mais, eu, pelo que eu tenho percebido." (TE13) 
A tentativa de suicídio possui múltiplas causas e envolve diferentes campos do conhecimento que se entrelaçam no esforço de compreendê-la (STORINO BD et al., 2018; JANUÁRIO JP e ÂNGELO MER, 2017). Dentre as questões relacionadas ao mundo interno, destacam-se nas falas dos entrevistados as alterações psicossociais da adolescência e sua relação com a tentativa de suicídio. As dificuldades próprias do ser adolescente foram abordadas por 04 entrevistados, destacando-se abaixo a fala de dois deles.

"Adolescência é uma fase da vida muito difícil né? Porque aparece um monte de coisa, um monte de dúvida na cabeça, o corpo tem mudança né? Você vai decidir sua opção sexual também nessa fase [...]." (TE6).

"A dificuldade em lidar com a temática da dualidade da infância com ser adulto [...]." (E20)

Os conflitos internos, a falta de resiliência e a necessidade de externalizar o não dito surge na fala dos entrevistados como possíveis causas para tentativa de suicídio. A incapacidade de lidar com conflitos emerge na fala de nove participante.

"O adolescente ele quer extravasar a dor que está dentro dele, e que ele não consegue superar naquele momento, e com isso, ele cai nessa situação." (TE10).

"[..]O adolescente não conseguir superar, né, as suas frustrações né, as suas decepções." (TE18)

"[...] a baixa tolerância da nova geração quanto as coisas negativas, eles não estão sabendo lidar com a intolerância, com as dificuldades do dia a dia." (E20)

Cerca de $20 \%$ dos participantes da pesquisa, enxergam, na tentativa de suicídio, uma maneira dos adolescentes resolverem seus conflitos e demonstrarem ao mundo sua insatisfação com sua situação de vida. Falas imbuídas de preconceito surgem neste momento e os adolescentes são caracterizados como "chamadores de atenção". Tal fato gera preocupação tendo em vista que a relação do profissional com o sujeito cuidado, pode influenciar de forma positiva ou negativa na procura por ajuda profissional no caso de novas tentativas de suicídio.

"Bom, o suicídio na adolescência se não for ligado a nenhum distúrbio de bipolaridade ou de transtorno mental ele vem acabar como uma chamativa de atenção mesmo, pro adolescente chamar atenção dos familiares, namorados essas coisas." (TE8).

"Na maioria das vezes é uma tendência de aparecer e também a falta de paz." (E23)

Sabe-se que $90 \%$ das pessoas que tentam suicídio possuem algum adoecimento mental (BOTTI NCL, et al., 2018). Apesar desta relevante informação, apenas um profissional correlacionou a tentativa de autoextermínio com a saúde mental.

\section{"[...] se não for ligado a nenhum distúrbio de bipolaridade ou de transtorno mental [...]." (TE8)}

Além das questões relacionadas ao mundo interno do adolescente, os profissionais de enfermagem entrevistados elencaram questões sociais como fatores preponderantes para que a tentativa de suicídio acontecesse, dentre as quais se destacou a falta de estrutura familiar, o uso de drogas, a desilusão amorosa e a falta de inserção religiosa. Estas questões foram elencadas por mais de 15 entrevistados.

"Eu acho que é mais questão familiar mesmo, conflito familiar." (TE17)

"Nos casos que já vi e que teve aqui com a gente, foi mesmo brigas, brigas de namorado [...] aí vem a questão das drogas, pelo que vi". (E5)

"[...] eu acho assim que as pessoas que tenta tirar a vida, ela tá muito sem direção espiritual, né?! Muito sem Deus, então eu acho que envolve isso, mas eu não quero culpar os pais, mas queria dizer que eles têm que tá mais atento a isso, sabe, direcionamento espiritual pra que o adolescente, cidadão tenha mais amor, né?!" (E12) 
A falta de suporte social, de uma rede social que acolha as demandas dos adolescentes e os permita sentirem-se inseridos em uma comunidade também apareceu nos relatos dos profissionais como possível causa para a tentativa de suicídio.

"Eu acho que hoje seria carência, assim, eu acho carência de tudo, sabe uma carência de educação, de saúde, de atenção da sociedade como um todo para esse adolescente, e isso leva à droga, leva a coisas depressivas que chega a uma tentativa de autoextermínio." (E21)

"[...] a base familiar é o começo de tudo, e depois também como esse adolescente é orientado na escola na parte social dele também." (TE 19)

\section{DISCUSSÃO}

Falar sobre suicídio é sempre um tabu, seja nas rodas de conversa, nos serviços de saúde, nas escolas e universidades. Quando se trata do suicídio em uma população específica como adolescentes, as dificuldades para discutir a temática são ainda maiores, pois é complexo compreender porque um adolescente, que está em uma fase de descobertas e de início de uma nova vida, busca a morte. Desta maneira, o reconhecimento ainda que de forma empírica sobre o aumento dos casos de suicídio entre adolescentes por parte dos entrevistados é um fator positivo, mas também preocupante. Tal identificação mostra que há uma sensibilidade sobre o tema abordado por parte dos entrevistados, mas também caminha ao encontro dos dados epidemiológicos mundiais e brasileiros que indica um aumento nas estatísticas de falecimento por suicídio.

Dados demonstram que cerca de 800.000 pessoas morrem por suicídio todos os anos no mundo, (WORLD HEALTH ORGANIZATION, 2019). Em relação aos adolescentes e jovens, estes dados são ainda mais alarmantes. Segundo o Mapa da Violência: Os jovens do Brasil, o maior crescimento de mortes por suicídio se deu na faixa etária de 15 a 19 anos, entre os anos 1990 e 2012. No ano de 2012 morreram por suicídio no país 117 adolescentes de 10 a 14 anos e 675 entre 15 e 19 anos. Ressalta-se que os números apresentados podem se encontrar subestimados devido ao registro incorreto de óbitos por suicídio, já que muitas das mortes acabam sendo classificadas como não intencional ou acidental (MOTA MA, 2018).

A manifestação do suicídio na adolescência associado a características próprias dessa fase foi evidenciada por quatro entrevistados. A adolescência é uma fase de profundas transformações biopsicossociais em que 0 adolescente buscará a construção de uma nova identidade e lutará por gradativa emancipação perante a família e escola. (ASSIS SG, et al., 2015).

As mudanças comportamentais do adolescente são tão desafiadoras que, por vezes, é difícil distinguir quando o adolescente chega a um limiar de dor e frustração com o qual não consegue lidar. Apesar disso, não se convém associar as tentativas de suicídio somente à crise na adolescência, pois o cuidado a este sujeito será sempre adiado, uma vez que se trata apenas de uma crise e portanto, passará, espontaneamente. Esta associação pode ser também uma maneira de negar o suicídio nesta faixa etária e postergar a sua discussão.

A ausência de resiliência também foi um ponto amplamente discutido pelos participantes. Sabe-se que a resiliência é um fator protetor contra episódios depressivos, seja o primeiro episódio ou sua recorrência. Estratégias para lidar com o estresse, desenvolver emoções positivas e aumentar a flexibilidade cognitiva, são pontos importantes para enfrentar agentes estressores, desenvolver a resiliência e, portanto, também reduzir as tentativas de suicídio (GANNAM SSA, 2018).

Ainda que o tempo de permanência na unidade de Pronto atendimento seja reduzido, há a possibilidade de estabelecer diálogo junto a este jovem. No momento da assistência, a enfermagem deve enxergar 0 adolescente para além do sujeito que tenta suicídio, uma vez que ele apresenta potencialidades a serem trabalhadas e que podem ser o ponto de partida para o desenvolvimento da resiliência. Assim como identificado na fala de cinco entrevistados, outras investigações apontam o suicídio, visto por profissionais de saúde, como uma tentativa de chamar a atenção (SILVA KFA, et al., 2016). 
Tal comportamento pode ocorrer por enxergarem no sujeito alguém que deseja ou que desejou ir na contra mão do objetivo de sua profissão, que é promover e manter a vida. Isso nos indica a necessidade de qualificar os profissionais de enfermagem para que os mesmos cuidem destes pacientes com compaixão, de maneira empática, mas sem tomar para si as dores que não Ihe pertencem (CESCON LF, et al., 2018; SILVA KFA, et al., 2016). A falta de conhecimento pode gerar no profissional de saúde, mais especificamente no enfermeiro, o desejo de fuga da assistência ao adolescente que busca o suicídio, até por não saber como agir, o que e como abordar.

No presente estudo foi interessante observar que apenas um profissional correlacionou a tentativa de suicídio na adolescência com o adoecimento mental. Conhecer as possíveis relações do suicídio com adoecimento mental permite ao enfermeiro atuar de forma a realizar os encaminhamentos necessários para que este adolescente não fique desassistido no momento em que receber alta da unidade de urgência. Há evidência na literatura que profissionais de saúde podem subestimar a necessidade de ajuda daqueles que tentam o autoextermínio por negligenciarem as causas que levam o indivíduo a tentar o ato (REISDORFER $\mathrm{N}$ et al.,2015).

Para mais de $50 \%$ dos participantes da pesquisa as tentativas de suicídio na adolescência também estão relacionadas a fatores sociais como desestruturação familiar, falta de apoio social, e uso e abuso de drogas. A literatura evidencia que tais fatores possuem forte impacto nas tentativas de suicídio, principalmente entre adolescentes (BARROS AMC, 2018; BARBOSA FNM et al., 2016).

Ressalta-se que adolescentes que se encontram em situação de vulnerabilidade social são aqueles que vivem negativamente as consequências das desigualdades sociais; da pobreza e da exclusão social; da falta de vínculos afetivos na família e nos demais espaços de socialização. Sofrem pela falta de acesso à educação, trabalho, saúde, lazer, alimentação e cultura. Tais fatores como esses influenciam negativamente no comportamento desses adolescentes e podem influenciar na tentativa de suicídio (BOTTI NCL, et al., 2018; MISSIAS-MOREIRA R, 2018).

Especificamente os conflitos no núcleo familiar afetam diretamente na autoestima de adolescentes e são considerados importantes fatores de risco na tentativa de suicídio e no suicídio. (BRÁS M et al., 2016; MAGNANI RM e STAUDT ACP, 2018). Assim como nos conflitos familiares, na relação amorosa a desilusão pode atuar como um gatilho emocional para a tentativa de suicídio. O término de um relacionamento com alguém significativo para o adolescente tem o poder de fazê-lo desejar romper com toda estrutura afetiva que tenha conquistado até então, e o suicídio surge como uma saída para o seu sofrimento (CZAPSKI ARS, et al., 2018).

Os participantes do estudo não citaram diretamente fatores que podem atuar na proteção dos adolescentes frente ao suicídio. Porém, sabe-se uma rede de apoio social e familiar são fundamentais nesta prevenção. Estas permitem ao adolescente o desenvolvimento de resiliência quando de sua passagem pelas experiências de desilusões e frustrações que a vida Ihe trará (BRÁS M et al., 2016).

Além da família e dispositivos sociais destaca-se a religiosidade como fator protetor. A participação ativa em atividades religiosas está fortemente associada a um comportamento saudável. Indivíduos que seguem uma religião, doutrina ou ensinamentos de sua fé, recebem mais orientações moral e prática com relação a como promover, conservar ou recuperar a saúde ou o bem-estar físico e emocional. Conhecer a vinculação do paciente com a religiosidade ou espiritualidade, permite ao profissional de enfermagem atuar para promover o conforto espiritual do paciente de maneira que este consiga enfrentar com maior resiliência a situação de agravo em que se encontra (LOUREIRO ACT, et al., 2015).

Neste campo, torna-se imprescindível aos profissionais de enfermagem que reconheçam a rede de assistência à saúde de sua região, atuem de maneira multiprofissional e consigam fazer o contra referenciamento deste adolescente para os outros dispositivos sociais e de saúde. A atuação em rede pode auxiliar na inserção deste adolescente em uma teia social que dê ao mesmo suporte físico e emocional ao adolescente. As ações intersetoriais com envolvimento de escolas, igrejas, empresas e organizações não governamentais são estratégias importantes para inserção do adolescente no meio social e ressignificação de sua existência. 
Os achados deste estudo, apesar de se limitarem à unidade de saúde pesquisada possivelmente refletem a realidade de outros serviços de saúde. É importante que a discussão acerca do suicídio na adolescência possa ser implantada em todos os espaços em que estes sujeitos estão inseridos.

\section{CONSIDERAÇÕES FINAIS}

A enfermagem reconhece o suicídio na adolescência como um problema atual e emergente. Correlacionam-no com características próprias da adolescência, mas também compreendem que fatores sociais e familiares influenciam na conduta suicida. Há dificuldade em associar o tema com adoecimento mental, o que pode influenciar na assistência prestada. Também chama atenção o fato da tentativa de suicídio na adolescência ser definida como uma forma de chamar a atenção de pais e sociedade. Percebe-se, portanto, a necessidade de educação permanente com profissionais que atuam diretamente com estas emergências. Espera-se que estes achados possam contribuir para o debate construtivo, visando cada vez mais um atendimento com embasamento científico e realizado com respeito, humanização e qualidade.

\section{REFERÊNCIAS}

1. ASSIS SG, et al. Adolescência e saúde coletiva: entre o risco e o protagonismo juvenil. Revista Ciência \&Saúde Coletiva, 2015;20 (11).

2. BARBOSA FNM, et al. Comportamento de risco à saúde de adolescentes escolares. Texto\&Contexto Enfermagem, 2016; 25 (4).

3. BARROS AMC. Suicídio entre jovens na escola: levantamento de fatores motivadores entre vítimas na literatura. Dissertação (Pós-Graduação em Saúde da Família) - Instituto de Ciências da Saúde, Universidade da Integração Internacional da Lusofonia Afro-Brasileira, Fortaleza, 2018; 29 p.

4. BRÁS M, et al. Fatores psicológicos de risco e protetores associados à ideação suicida em adolescentes. Revista Psicologia, Saúde\& Doenças, 2016; 17 (2): 132-149.

5. BOTTI NCL, et al. Tentativa de suicídio entre pessoas com transtornos mentais e comportamentais. Revista de Enfermagem UFPE online, 2018;12 (5): 1289-1295.

6. CESCON LF, et al. Aproximações e distanciamentos ao suicídio: analisadores de um serviço de atenção psicossocial. Saúde e Sociedade, 2018;27 (1).

7. CZAPSKI ARS, et al. Saúde mental vigilância, prevenção e atenção ao suicídio. Revista Extensão, $2018 ; 2$ (1).

8. GANNAM SSA. O mal-estar na formação médica: uma análise dos sintomas de ansiedade, depressão e esgotamento profissional e suas relações com resiliência e empatia. Dissertação (Doutorado em Ciências) - Faculdade de Medicina, Universidade de São Paulo, São Paulo, 2018; 122 p.

9. JANUÁRIO JP, ÂNGELO MER. Abordagem do profissional de enfermagem em indivíduos com comportamento suicida: revisão integrativa - 2006 a 2016. Dissertação (Graduação em Enfermagem)- Centro Universitário São Lucas, Porto Velho, 2017; 26 p.

10. LOUREIRO ACT, et al. Espiritualidade como fator de proteção do suicídio. Revista Brasileira de Psicologia, 2015;2 (2): 33-40.

11. MAGNANI RM, STAUDT ACP. Estilos parentais e suicídio na adolescência: uma reflexão acerca dos fatores de proteção. Pensando famílias, 2018;22 (1): 75-86.

12. MISSIAS-MOREIRA R. Condições de saúde, qualidade de vida e comportamentos de risco de adolescentes. Relicário Revista do MAS, 2018;5 (10).

13. MORAES SM, et al. Atitudes relacionadas ao suicídio entre graduandos de enfermagem e fatores associados. Acta Paulista de Enfermagem, 2016;29(6):643-649.

14. MOTA MA. Comportamento suicida em adolescentes: uma revisão de literatura. Dissertação (Graduação em Enfermagem) - Faculdade de Ciências da Educação e Saúde, Centro Universitário de Brasília, Brasília, $2018 ; 17$ p.

15. PALANGANA IC. Desenvolvimento e aprendizagem em Piaget e Vygotsky: a relevância do social. 6 ed. São Paulo: Summus Editorial, 2015; $176 \mathrm{p}$.

16. REISDORFER N, et al. Suicídio na voz de profissionais de enfermagem e estratégias de intervenção diante do comportamento suicida. Revista de Enfermagem UFSM, 2015;5 (2): 295-304.

17. SANTOS RS, et al. Nurses' actions towards suicide attempters: reflective analysis. Revista de Enfermagem UFPE online, 2017;11(2):742-748.

18. SILVA KFA, et al. Suicídio: uma escolha existencial frente ao desespero humano. Pretextos Revista da Graduação em Psicologia da PUC Minas, 2016;1 (2).

19. SOUZA ACG, et al. Suicídio na adolescência: revisão da literatura. Revista Uningá, 2015; 43:95-98.

20. STORINO BD, et al. Atitudes de profissionais de saúde em relação ao comportamento suicida. Cadernos de Saúde Coletiva, 2018; 26 (4): 369-377.

21. WORLD HEALTH ORGANIZATION. 2019.Suicide in the world: global health estimates. In:World Health Organization.Disponível em: https://apps.who.int/iris/bitstream/handle/10665/326948/WHO-MSD-MER-19.3eng.pdf?sequence=1\&isAllowed=y. Acesso em: 1 mar. 2020. 